\title{
Implementation of four-point fully implicit block method for solving ordinary differential equations
}

\begin{abstract}
This paper describes the development of a four-point fully implicit block method for solving first order ordinary differential equations (ODEs) using variable step size. This method will estimate the solutions of initial value problems (IVPs) at four points simultaneously. The method developed is suitable for the numerical integration of non-stiff and mildly stiff differential systems. The performances of the four-point block method are compared in terms of maximum error, total number of steps and execution times to the non-block method 1PVSO in [Z. Omar, Developing parallel block methods for solving higher order ODEs directly, Ph.D. Thesis, University Putra Malaysia, Malaysia, 1999].
\end{abstract}

Keyword: Four-point,Implicit block method,Block method,Ordinary differential equations 